\title{
Refolding by High Pressure of a Toxin Containing Seven Disulfide Bonds: Bothropstoxin-1 from Bothrops jararacussu
}

\author{
Keli N. Balduino $\cdot$ Patrick J. Spencer • \\ Natalia V. Malavasi · Rosa M. Chura-Chambi • \\ Laura S. Lemke • Ligia Morganti
}

Published online: 22 December 2010

(c) The Author(s) 2010. This article is published with open access at Springerlink.com

\begin{abstract}
Aggregation is a serious obstacle for recovery of biologically active heterologous proteins from inclusion bodies (IBs) produced by recombinant bacteria. E. coli transformed with a vector containing the cDNA for Bothropstoxin-1 (BthTx-1) expressed the recombinant product as IBs. In order to obtain the native toxin, insoluble and aggregated protein was refolded using high hydrostatic pressure (HHP). IBs were dissolved and refolded ( $2 \mathrm{kbar}$, $16 \mathrm{~h}$ ), and the effects of protein concentration, as well as changes in ratio and concentration of oxido-shuffling reagents, guanidine hydrochloride $(\mathrm{GdnHCl})$, and $\mathrm{pH}$ in the refolding buffer, were assayed. A $32 \%$ yield $(7.6 \mathrm{mg}$ per liter of bacterial culture) in refolding of the native BthTx-1 was obtained using optimal conditions of the refolding buffer (Tris- $\mathrm{HCl}$ buffer, $\mathrm{pH} 7.5$, containing $3 \mathrm{mM}$ of a 2:3 ratio of GSH/GSSG, and $1 \mathrm{M} \mathrm{GdnHCl).} \mathrm{Scanning} \mathrm{electron}$ microscopy (SEM) showed that that disaggregation of part of IBs particles occurred upon compression and that the morphology of the remaining IBs, spherical particles, was not substantially altered. Dose-dependent cytotoxic activity of high-pressure refolded BthTx-1 was shown in C2C12 muscle cells.
\end{abstract}

Keywords High hydrostatic pressure - Refolding · Myotoxin $\cdot$ K49 phospholipase $\cdot$ Aggregation

\section{Abbreviations \\ BthTx-1 Bothropstoxin-1}

K. N. Balduino - P. J. Spencer · N. V. Malavasi ·

R. M. Chura-Chambi · L. S. Lemke · L. Morganti ( $\square)$

Centro de Biotecnologia, Instituto de Pesquisas Energéticas e

Nucleares-CNEN/SP, Av. Professor Lineu Prestes, 2242,

São Paulo, SP 05508-000, Brazil

e-mail: 1morganti@ipen.br
GdnHCl Guanidine hydrochloride

SEM Scanning electron microscopy

$\mathrm{PLA}_{2}$ Phospholipase $\mathrm{A}_{2}$

GSSG Oxidized glutathione

GSH Reduced glutathione

\section{Introduction}

Myotoxins are widespread components in venoms of snakes from different genera. They are responsible for intense disruption of muscle cells in tissues adjacent to the bite site, thus contributing to the severity of accidents and permanent sequelae that may occur in the most severe cases [1]. These toxins belong to two major structural groups. One that includes short-chain peptides, such as crotamine and myotoxin A [2], while the other is composed of molecules with phospholipase $\mathrm{A}_{2}\left(\mathrm{PLA}_{2}\right)$ structure [3]. Toxins of the latter group display structural motifs typical of mammalian inflammatory $\mathrm{PLA}_{2} \mathrm{~s}$, in which 14 cysteine residues responsible for the assembly of seven disulfide bonds are conserved and the scaffold is highly preserved [4]. In addition to myotoxic activity, snake venom $\mathrm{PLA}_{2} \mathrm{~S}$ may present pharmacological activities with anticoagulant, neurotoxic, or cardiotoxic properties [5]. Many myotoxins in this group are almost inactive when assayed in the usual mixed-micelle enzyme tests because they display critical substitutions within the calcium-binding loop [6]. These include Q4/E, Y28/Q, and D49/K mutations [7]. Toxins bearing these features are commonly referred to as K49 myotoxins. Although K49 toxins are enzymatically inactive, they induce severe myonecrosis. Their activities are comparable to their catalytically active counterparts [8] by 
a mechanism not yet fully understood. In order to further characterize these toxins and their mechanisms of action, studies using site-directed mutagenesis would be helpful. However, expression of biologically active recombinant K49 myotoxins is a challenging task, especially because of the high number of disulfide bonds, and solubilization and refolding of such proteins from IBs using classical refolding protocols usually result in low yields.

In traditional techniques for protein refolding, high concentrations of chaotropic agents, such as $\mathrm{GdnHCl}$ and urea, are used to solubilize recombinant IBs. Unfolded proteins are then refolded by reduction in the concentration of the chemical denaturants either by dilution or by chromatographic procedures, such as size-exclusion chromatography, on-column or immobilized chaperone-assisted refolding [9]. However, these conventional refolding methods frequently allow generation of new aggregates during the process of chaotropic agent removal, resulting in low levels of protein in the soluble and native states.

The ability of high hydrostatic pressure (HHP) to dissociate oligomeric proteins was theoretically predicted and experimentally confirmed [10]. High pressure acts on protein structure favoring molecular transitions that reduce system volume. Specific volumes in aggregated proteins are larger than in native states due to the presence of intermolecular water-excluded cavities. HHP in the range 1-3 kbar disfavor intermolecular hydrophobic and electrostatic interactions leading to protein disaggregation [11-13]. The same types of interaction are also responsible for protein structure in the native states. However, typically proteins do not denature until pressures above $5 \mathrm{kbar}$ at room temperature are reached. Thus, dissociation of macromolecular complexes by HHP is not accompanied by looseness of the existing secondary and tertiary structures of the dissociated states. In the preceding decade, dissolution and refolding of some recombinant proteins produced as IBs in E. coli [14-19] and aggregated proteins [20-22] were described.

Refolding of the toxin Bothropstoxin-1 (BthTx-1), a small protein $(13.7 \mathrm{kDa})$ containing seven disulfide bridges, was previously obtained by solubilization of IBs under denaturing conditions followed by size-exclusion chromatography in the presence of surfactants with a $2.5 \%$ yield of the native form [23].

Due to the high amount of cysteine residues in the native BthTx-1 molecule, it is unavoidable that structures with many intra- and inter-chain bond combinations arise with the use of conventional methods to refold denatured BthTx-1. In such condition, obtaining properly refolded molecules, with correct disulfide bonds and toxic activity, is a rather difficult task to be achieved, and this makes BthTx-1 refolding a challenging objective.

\section{Materials and Methods}

\section{BthTx-1 Expression and IB Preparation}

The expression plasmid pET24-BthTX-1, which contains codons for translation of a protein with a Met residue, followed by the sequence of BthTx-1, was transformed by electroporation in competent E. coli BL21 cells. For BthTx-1 expression, a culture was carried out in a $1000 \mathrm{ml}$ shake flask containing $250 \mathrm{ml}$ of rich culture medium (2X-HKSII) supplemented with kanamycin $(50 \mu \mathrm{g} / \mathrm{ml})$. The flasks were inoculated with a pre-cultivated medium $(20 \mathrm{ml})$ containing $E$. coli harboring the expression plasmid and incubated $\left(37^{\circ} \mathrm{C}\right)$ in a rotary shaker until optical density $\left(\mathrm{OD}_{600 \mathrm{~nm}}\right)$ of the culture reached the value of 3.0. Protein expression was then induced with addition of isopropyl $\beta$-D-thiogalactopyranoside IPTG $(0.5 \mathrm{mM}$, final concentration) and the cultures were grown for 16 additional hours and harvested by centrifugation. The pellets were resuspended in Tris- $\mathrm{HCl}(50 \mathrm{mM}, 50 \mathrm{ml}, \mathrm{pH} 7.5)$, containing EDTA $(2 \mathrm{mM})$, Triton X-100 $(0.1 \%$, v/v) and $50 \mu \mathrm{g} / \mathrm{ml}$ lysozyme and incubated at $25^{\circ} \mathrm{C}$ for $15 \mathrm{~min}$. The suspension was sonicated and centrifuged $\left(8,000 \times g, 4^{\circ} \mathrm{C}\right.$, $10 \mathrm{~min})$. The supernatant was discarded; the pellet was resuspended in $50 \mathrm{ml}$ Tris- $\mathrm{HCl}(50 \mathrm{mM}, \mathrm{pH} 7.5)$, EDTA $(2 \mathrm{mM})$, and Triton X-100 $(0.1 \%, \mathrm{v} / \mathrm{v})$. The centrifugation and resuspension steps were repeated five times; the resulting pellet was dissolved in Tris- $\mathrm{HCl}(50 \mathrm{mM}, \mathrm{pH}$ $7.5)$, resuspended in the same buffer and stored $\left(-20^{\circ} \mathrm{C}\right)$ for further assays.

\section{Formulation of Refolding Buffers and Sample Compression Assays}

Suspensions of IBs were diluted in refolding buffer (50 mM Tris- $\mathrm{HCl}, \mathrm{pH} 7.5,1 \mathrm{mM}$ EDTA) containing different proportions of GSH/GSSG and concentrations of the redox pair, $\mathrm{GdnHCl}$ or different $\mathrm{pHs}$, as indicated in Table 1 and Fig. 1. Suspension samples $(1.0 \mathrm{ml})$ were placed into plastic bags, which were sealed and placed into a larger plastic bag, which was also sealed under vacuum. This bag was then placed into a pressure vessel (High Pressure Equipment, Model R4-6-40) filled with a mixture of water and oil as pressure-transmitting fluid and, high pressure $(2 \mathrm{kbar})$ was applied $(16 \mathrm{~h})$ using an air compressor fitted to the system (High Pressure Equipment, Model PS-40). After decompression, the samples were centrifuged $(12,000 \times g, 15 \mathrm{~min})$ to remove the insoluble aggregates. The supernatants were dialyzed, centrifuged again to remove insoluble aggregates formed during dialysis and stored $\left(-20^{\circ} \mathrm{C}\right)$ for later analysis. All assays were performed at least twice. 
Table 1 Analysis of the soluble BthTx-1 obtained by pressurization of IBs suspensions

\begin{tabular}{|c|c|}
\hline & Soluble protein recovery $(\%)^{\mathrm{a}}$ \\
\hline \multicolumn{2}{|c|}{ GSH:GSSG proportion } \\
\hline $1: 9$ & n.d. \\
\hline $1: 4$ & 4.4 \\
\hline $2: 3$ & 7.0 \\
\hline $1: 2$ & 2.5 \\
\hline $1: 1$ & n.d. \\
\hline $2: 1$ & n.d. \\
\hline $3: 2$ & n.d. \\
\hline $4: 1$ & n.d. \\
\hline $9: 1$ & n.d. \\
\hline \multicolumn{2}{|c|}{ 2GSH:3GSSG concentration (mM) } \\
\hline 0 & n.d. \\
\hline 0.7 & n.d. \\
\hline 1.5 & n.d. \\
\hline 3.0 & 9.6 \\
\hline 6.0 & 7.2 \\
\hline 10.0 & n.d. \\
\hline 20.0 & n.d. \\
\hline \multicolumn{2}{|l|}{$\mathrm{pH}$} \\
\hline 5.5 & 21.2 \\
\hline 6.5 & 12.5 \\
\hline 7.5 & 18.2 \\
\hline 8.5 & 5.0 \\
\hline 9.0 & n.d. \\
\hline \multicolumn{2}{|c|}{ GndHCl concentration $(\mathrm{M})$} \\
\hline 0 & n.d. \\
\hline 0.25 & n.d. \\
\hline 0.5 & n.d. \\
\hline 0.75 & n.d. \\
\hline 1 & 9.7 \\
\hline 2 & 14.5 \\
\hline 4 & 13.2 \\
\hline 6 & n.d. \\
\hline \multicolumn{2}{|c|}{ IBs optical density (600 nm) } \\
\hline 0.5 & 33.6 \\
\hline 1.0 & 24.6 \\
\hline 2.0 & 14.0 \\
\hline 4.0 & n.d. \\
\hline 6.0 & n.d. \\
\hline 8.0 & n.d. \\
\hline
\end{tabular}

Quantification of SDS-PAGE stained bands. Effect of glutathione redox pair ratios: Refolding buffer $\mathrm{pH} 7.5$ containing $2 \mathrm{M} \mathrm{GdnHCl}$ and $10 \mathrm{mM}$ GSH/GSSG; Effect of concentration of GSH/GSSG: Refolding buffer $\mathrm{pH}$ 7.5 containing $2 \mathrm{M} \mathrm{GdnHCl}$ and a 2GSH:3GSSG ratio; Effect of pHs: Refolding buffer, MES for $\mathrm{pHs}$ up to 6.5 and Tris-HCl for $\mathrm{pHs} 7.5$ and higher, containing $2 \mathrm{M} \mathrm{GdnHCl}$ and $3 \mathrm{mM}$ glutathione at a 2GSH:3GSSG ratio; Effect of concentration of GdnHCl: Refolding buffer, $\mathrm{pH} 7.5$, containing $3 \mathrm{mM}$ glutathione at a $2 \mathrm{GSH}: 3 \mathrm{GSSG}$ ratio; Effect of $\mathrm{OD}_{600 \mathrm{~nm}}$ readings: Refolding buffer, pH 7.5 containing $1 \mathrm{M} \mathrm{GdnHCl}$ and $3 \mathrm{mM}$ glutathione at a $2 \mathrm{GSH}$ :3GSSG ratio

n.d. non-detected

${ }^{a}$ The intensity of BthTX-1 bands was determined by digital densitometry analysis

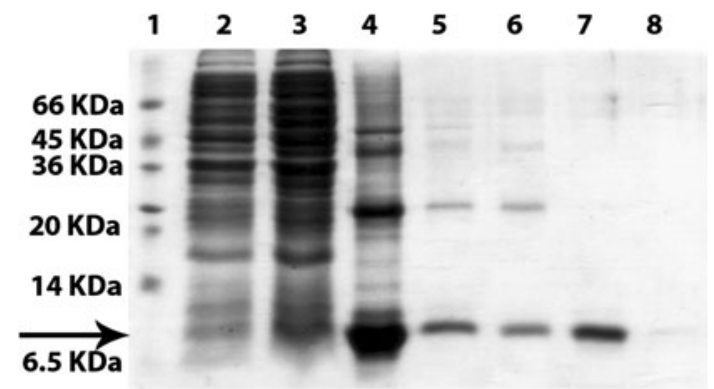

Fig. 1 Gel electrophoresis image showing the process phases, from the raw inclusion bodies to the final soluble active protein using refolding buffer containing $3 \mathrm{mM}$ glutathione at a $2: 3$ ratio of the redox pair GSH/GSSG with IBs at $0.5 \mathrm{OD}_{600 \mathrm{~nm}}$. Lane 1: molecular mass marker; Lane 2: total bacteria before induction; Lane 3: total bacteria after induction with isopropyl $\beta$-D-thiogalactopyranoside (IPTG); Lane 4: IBs (insoluble fraction) before compression; Lane 5: IBs (insoluble fraction) after compression; Lane 6: insoluble fraction formed after compression and dialysis; Lane 7: soluble fraction after compression and dialysis; Lane 8: soluble fraction after maintenance at atmospheric pressure and dialysis. Arrow indicates molecular mass of BthTX-1 monomer

\section{Protein Quantification}

Insoluble (IBs) and soluble BthTx-1 were diluted in Tris$\mathrm{HCl}$ (50 mM, pH 7.5) buffer containing urea (8 M) at appropriate ratios, and submitted in duplicate to total protein determination (Bradford assay). An absorbance curve $\left(A_{\mathrm{nm}}\right)$ for pure bovine serum albumin (BSA; $15-500 \mu \mathrm{g} / \mathrm{ml}$; Bradford assay) samples was used as a standard. The same samples were also submitted to both SDS-PAGE and staining with Coomassie blue. Digital densitometry of BthTx-1 bands was determined using the Image $\mathrm{J}$ software.

\section{Scanning Electron Microscopy}

Scanning electron microscopy (SEM) was performed after drying water-dialyzed insoluble aggregates (IB or the nonrefolded aggregates) onto clean polished Philips stubs and sputter-coated in a sputter coater (Balzer, model SCD-040, $38 \mathrm{~mA}, 120 \mathrm{~s})$. The samples were then examined and photographed in a Field Emission Scanning Electron Microscope (JEOL, JSM 670-1F). Size analysis of IBs was performed using the Image Tools software.

\section{Cytotoxic Activity}

C2C12 (ATCC CRL-1772), a murine skeletal myoblast cell line which can fuse and differentiate into myotubes, was grown in Dulbecco's Modified Eagle's Medium (DMEM; Sigma D-5796) supplemented with $10 \%$ fetal calf serum, $2 \mathrm{mM}$ glutamine, $1 \mathrm{mM}$ pyruvic acid, penicillin (100 U/ $\mathrm{ml})$, streptomycin $(0.1 \mathrm{mg} / \mathrm{ml})$, and amphotericin B $(0.25 \mu \mathrm{g} / \mathrm{ml})$, in a humified atmosphere with $5 \% \mathrm{CO}_{2}$, at 
$37^{\circ} \mathrm{C}$. C2C12 cells were seeded in 96-well microplates, at an approximate initial density of $1-4 \times 10^{3}$ cells per well. After reaching near confluence, usually in 3-5 days, medium was replaced by differentiation medium, which consisted of DMEM supplemented with 1\% FCS. After 4-6 additional days of culture, cells were incubated with BthTX-1 purified from crude venom, used as a control or recombinant BthTx-1 refolded at high pressure, diluted in a total of $250 \mu \mathrm{l}$ culture medium/well. After $4 \mathrm{~h}$ incubation, aliquots of the supernatant were collected and LDH activity was determined using a commercial kit (LDH LiquiformLabtest) and expressed as percentage of LDH release [24]. Reference control for $100 \% \mathrm{LDH}$ release consisted of supernatant of cells incubated with medium from cells incubated with $0.1 \%(\mathrm{v} / \mathrm{v})$ of Triton X-100. The assays were carried out in triplicates.

\section{Results}

Effect of Refolding Buffer on the Solubilization of BthTx-1 from IBs

The extraordinarily high number of free cysteine thiol groups present in the native BthTx-1 molecule was regarded as an indication that refolding of this protein could be complicated by the presence of non-native intra- and interprotein covalent disulfide bonds in IBs. Previous works reported that HHP in combination with oxido-shuffling agents, such as glutathione, can effectively refold covalently cross-linked aggregates of recombinant proteins produced as IBs in $E$. coli $[16,20]$. In order to evaluate the potential benefit of this approach, suspensions of IBs were incubated (2 kbar) in refolding buffer $(50 \mathrm{mM}$ Tris- $\mathrm{HCl}$, $\mathrm{pH} 7.5 ; 2 \mathrm{M} \mathrm{GdnHCl}$ ) containing varying ratios of reduced (GSH) and oxidized (GSSG) glutathione molecules at a $10 \mathrm{mM}$ final concentration. Presence of BthTx-1 in the supernatants after compression followed by dialysis and centrifugation, performed to withdraw the denaturing reagent and insoluble aggregates eventually formed during dialysis, was analyzed by SDS-PAGE (Table 1) and quantification of the bands of protein with a molecular mass of about $14 \mathrm{kDa}$, corresponding to BthTx-1. The sample of HHP-treated BthTx-1 exhibiting the most intense band, was obtained with a 2:3 ratio of the GSH:GSSG redox pair. For a fixed 2 GSH:3 GSSG proportion, $3 \mathrm{mM}$ proved to be the total molar concentration of the redox mixture in which the highest level of soluble BthTX-1 was obtained for pressure-induced refolding. Low (non-detectable) yields of soluble BthTx-1 was observed for supernatant of suspensions of IBs incubated at HHP in the absence of the redox pair, indicating that these reagents are essential for BthTx-1 refolding.
Incubation of BthTx-1 (2 kbar, $16 \mathrm{~h}$ ) was performed in the presence of refolding buffer at various pHs (5.5-9.0) and $\mathrm{pH}$ of 7.5 was chosen. A low value of intensity of band was obtained at the sample compressed at $\mathrm{pH} 8.5$, probably due to protein precipitation at this $\mathrm{pH}$ near the $\mathrm{p} I$ value for BthTx-1 (pI = 8.2).

Presence of additives at concentrations usually reported in the literature for protein refolding [25] (0.1\% polyethylene glycol 6000, 0.5 M arginine, 2.5 M glycerol, $1 \mathrm{M}$ sucrose, $1 \mathrm{M}$ glucose, $0.1 \mathrm{M}$ Tween-20, $0.5 \mathrm{mM}$ Triton $\mathrm{X}-100$, and $0.15 \mathrm{M} \mathrm{NaCl}$ ) did not improve the yields of soluble BthTx-1 (data not shown).

Presence of non-denaturing levels of $\mathrm{GdnHCl}$ was previously shown to improve the yields of refolding of aggregated proteins subjected to HHP. Pressurized BthTx1 IBs were not solubilized in the absence of $\mathrm{GdnHCl}$, as can be seen in the analysis of the SDS-PAGE bands (Table 1). Higher yields of soluble BthTx-1 were obtained in the presence of 2 and $4 \mathrm{M} \mathrm{GdnHCl}$. The band of BtxTh1 corresponding to the sample compressed in the presence of $1 \mathrm{M} \mathrm{GdnHCl}$ presented a slightly lower intensity. The lowest concentration ( $1 \mathrm{M}$ ) was used in the following experiments due to a higher probability of maintaining the secondary and tertiary native structures of BtxTh-1, which were previously shown to exist in IBs produced by $E$. coli in the absence or in presence of low concentration of denaturing reagent $[26,27]$. At $6 \mathrm{M} \mathrm{GdnHCl}$ the yield of soluble BthTx-1 diminishes, indicating that the protein was solubilized but did not fold correctly, thus being more prone to aggregation during dialysis.

The concentration of the aggregated protein can also be an important parameter, since the correct refolding pathway competes with the misfolding and aggregation ones. Usually, low protein concentrations (typically 50-100 $\mu \mathrm{g}$ / $\mathrm{ml}$ ) are required to prevent reaggregation and thus higher yields of refolded protein are obtained when traditional, chemically induced solubilization of aggregated proteins are used in refolding. Table 1 shows that the yields of soluble BthTx-1 obtained after compression (2 kbar) and dialysis were proportional to the dilutions of IBs. Thus, we choose a low BthTX-1 concentration (O.D. of 0.5), which corresponds to a protein concentration of approximately $50 \mu \mathrm{g} / \mathrm{ml}$, for the HHP refolding.

A SDS-PAGE image showing the phases in the process of obtaining soluble BthTx-1 is shown in Fig. 1. Expression of BthTx-1 by activated $E$. coli cells is low (lane 3 ). However, enrichment of insoluble BthTx-1 occurred after the cells were sonicated and IBs were washed, as shown in lane 4 . The sample treated with compression at optimal conditions is shown in lane 7 as a very strong and nearly unique band. As can be observed in lane 8 (sample maintained at atmospheric pressure), the BthTx-1 band is very faint, indicating that the protein was not solubilized/refolded under this condition. 
Fig. 2 Field scanning electron microscopy (SEM) of insoluble BthTx-1. a IBs; b insoluble aggregates after compression. Scale bars: $10 \mu \mathrm{m}$ (original magnification $\times 1000), 1 \mu \mathrm{m}$ (original magnification $\times 10,000)$

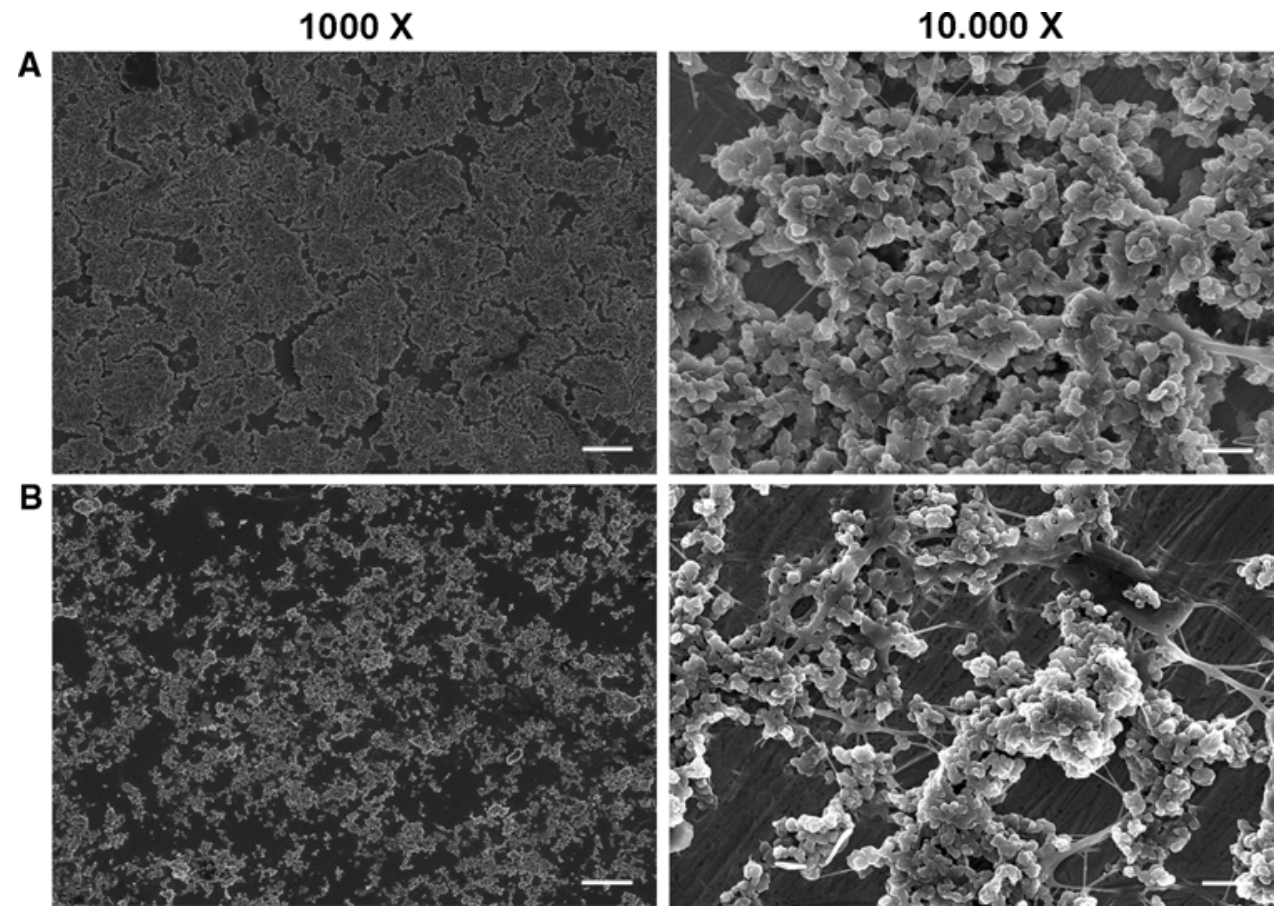

The yield of BthTx-1 solubilization was $32 \%$. A total amount of $7.6 \mathrm{mg}$ of BthTx-1 was obtained from 11 of bacterial culture.

The immunological identity of BthTx-1 was confirmed by western blot analysis (not shown).

Visualization of IBs and Insoluble Aggregates

Insoluble samples of BthTx-1, non-treated (A) or treated (B) with $2 \mathrm{kbar}$ pressure $(16 \mathrm{~h})$ in refolding buffer $(50 \mathrm{mM}$ Tris-HCl, pH 7.5, $1 \mathrm{mM}$ EDTA, $3 \mathrm{mM}$ glutathione, 2:3 ratio of $\mathrm{GSH} / \mathrm{GSSG}$, and $1 \mathrm{M} \mathrm{GdnHCl}$ ), are shown in Fig. 2. The IBs were mostly regular spherical particles with an average diameter of $0.27 \pm 0.032 \mu \mathrm{m}$. Although less dense material was observed, the morphology and size of the insoluble particles remaining after 2.0 kbar application was similar to the non-treated IBs, suggesting that the high pressure was not able to induce solubilization of all the IBs particles.

\section{Biological Activity Determination}

BthTx-1 is a myotoxic protein, which shows lytic activity on differentiated muscle cells [24]. BthTx-1 cytotoxicity was estimated by the release of LDH to supernatant after incubation with the toxin for $4 \mathrm{~h}$. Figure 3 shows LDH activity in the supernatant of $\mathrm{C} 2 \mathrm{C} 12$ muscle cells treated with the refolded toxin and with the natural one, purified from $B$. jararacussu venom. The cytotoxic effect induced by the recombinant protein at the concentration of $30-120 \mu \mathrm{g} / \mathrm{ml}$ was comparable to the activity of the natural

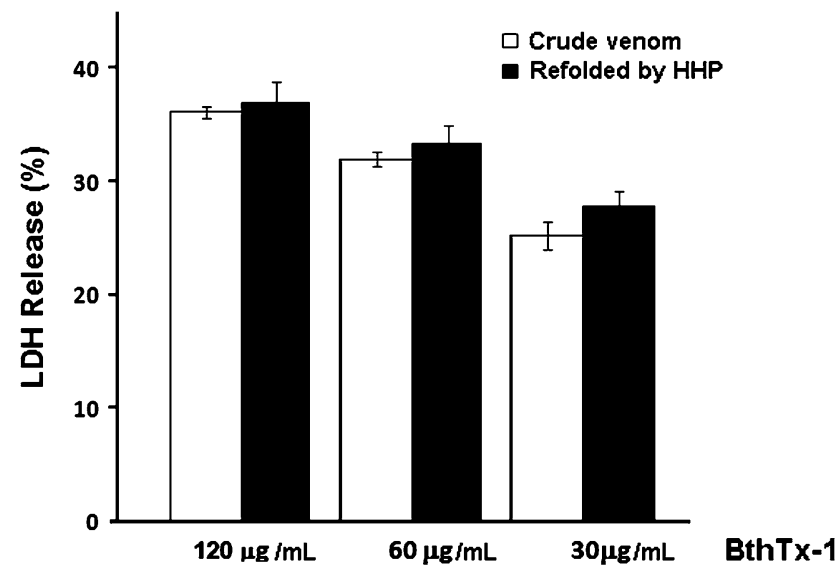

Fig. 3 Cytotoxicity was determined by the release of LDH to cell supernatants, $4 \mathrm{~h}$ after exposure of the cells to recombinant BthTx-1 refolded under high pressure or to BthTx-1from crude venom. Each bar represents mean $\pm \mathrm{SD}$ of triplicate cultures

toxin thus validating our refolding procedure. Dosedependent cytolytic activity of BthTx-1 refolded under HHP is shown for $\mathrm{C} 2 \mathrm{C} 12$ cells.

\section{Discussion}

Our strategy for expression of BthTx-1 resulted in production of this toxin within IBs, which were easily separated from soluble bacterial proteins by centrifugation.

Starting from IBs, dissociation of the insoluble aggregates was a problem to be solved. In order to obtain protein refolding, traditional approaches use high concentrations of 
chemical denaturing reagents for disaggregation that also results in essentially complete unfolding of protein molecules. Thus, interactions between non-native protein molecules occur during removal of the denaturing reagent and strong re-aggregation is usually observed [5]. Together with misfolded states, native proteins with secondary and even certain amount of tertiary structures were shown in bacterial recombinant IBs [26, 27]. It has also been shown that use of mild conditions for solubilization of IBs favors maintaining native structures [18, 28, 29], thus precluding re-aggregation. Dissolution of covalently cross-linked aggregates requires disruption of non-native hydrophobic contacts, electrostatic interactions, and hydrogen and disulfide bonds. HHP has been reported to disrupt both ionic and hydrophobic interactions. However, hydrogen bonds are pressure insensitive [13] and non-native hydrogen bonds among aggregated molecules can interfere with pressure-induced dissolution. It has already been shown that presence of low concentrations of $\mathrm{GdnHCl}$ can favor disruption of those bonds allowing dissolution of aggregated states [18]. Since HHP favors protein disaggregation, we solubilized BthTx-1 under a non-denaturing condition and low concentration (1 M) of $\mathrm{GdnHCl}$, a condition milder than that described for solubilization of BthTx-1 IBs under atmospheric pressure and denaturing conditions [23].

Usually, high number of disulfide bonds makes refolding procedures difficult. BthTx-1 contains seven disulfide bonds [30]. Addition of redox shuffling agents to covalently cross-linked aggregates facilitates refolding of disulfidecontaining proteins by breaking non-native intermolecular disulfide bonds under conditions that allow these bonds to be formed [19]. As expected, both the redox pair GSH/ GSSG ratio and glutathione concentration were shown to be essential for the recovery of BthTx-1 in the refolding process. We found that the optimal ratio and concentration for these reagents were $2 \mathrm{GSH}: 3 \mathrm{GSSG}$ and $3 \mathrm{mM}$, respectively. The $\mathrm{pH}$ value of 7.5 was chosen for HHP-driven dissolution and refolding of BthTx-1.

The effects of additives on refolding yields of native proteins have been tested with traditional refolding protocols. Although the mechanism of action of these compounds is not well understood, empirical screening of dissolution additives occasionally led to formulations that substantially increase the refolding yield at atmospheric pressure [31, 32]. The effect of the presence of some additives on HHP-refolding has also been tested. Arginine $(0.5 \mathrm{M})$ combined with HHP was most effective to obtain three functional Gram-negative binding proteins (GNBP1, GNBP2, and GNBP3) and two human phosphatases from IBs [21]. In this study, none of the additives tested further enhanced the yields of refolded BthTx-1.

Although BthTx-1 3-D structure is identical to that of $\mathrm{PLA}_{2}$, this protein is unable to bind $\mathrm{Ca}^{2+}$ due to a D49/K mutation and is therefore devoid of the catalytic activity characteristic of this class of enzyme. For this reason, phospholipase activity was not used to determine BthTx-1 bioactivity. LDH detection assays in myoblasts or myotubule cultures treated with BthTx-1 were performed. Our results showed that the protein refolded under pressure exhibit cytolytic activity, indicating that the treatment led to the formation of BthTx-1 with biofunctional structure.

In conclusion, solubilization and refolding of high levels of biologically active BthTx-1 was achieved using HHP. We obtained $32 \%$ of BthTx-1 refolding, a yield much higher than that previously described (2.5\%) [23], in which denaturing conditions were used for solubilization of IBs.

Several authors [33-36] have expressed recombinant snake $\mathrm{PLA}_{2} \mathrm{~s}$, mostly aiming the obtention of mutants for structure-function studies. However, in most cases, the yields of properly refolded proteins, using classical protocols were fairly low, fact that might be ascribed to the 7 disulfide bonds that reticulate the toxin and might form intra and inter-molecular bonds during the refolding process. We believe that our approach might represent an attractive alternative to circumvent this difficulty, a milestone for future site-directed mutagenesis studies, which are so necessary for further understanding the mechanism of action of these toxins.

Acknowledgments This work was supported by grants from the State of São Paulo Research Foundation-FAPESP (Process 07/54624-4) and National Council for Scientific and Technological Development-CNPq (Process 479816/2007-7). The authors acknowledge Dr Paulo Boschcov, former professor at UNIFESP, whose suggestions contributed to improve the quality of the final version of the manuscript and Centro de Ciência e Tecnologia dos Materiais/IPEN for the Scanning Electron Microscope analysis.

Open Access This article is distributed under the terms of the Creative Commons Attribution Noncommercial License which permits any noncommercial use, distribution, and reproduction in any medium, provided the original author(s) and source are credited.

\section{References}

1. Gutierrez, J. M., \& Lomonte, B. (1995). Phospholipase a(2), myotoxins from bothrops snake-venoms. Toxicon, 33, 14051424.

2. Bober, M. A., Glenn, J. L., Straight, R. C., \& Ownby, C. L. (1988). Detection of myotoxin alpha-like proteins in various snake venoms. Toxicon, 26, 665-673.

3. Fletcher, J. E., \& Jiang, M. S. (1998). Lys49 phospholipase A(2) myotoxins lyse cell cultures by two distinct mechanisms. Toxicon, 36, 1549-1555.

4. Pungercar, J., Krizaj, I., Liang, N. S., \& Gubensek, F. (1999). An aromatic, but not a basic, residue is involved in the toxicity of group-II phospholipase A(2) neurotoxins. Biochemical Journal, 341, 139-145

5. Wang, Y. M., Lu, P. J., Ho, C. L., \& Tsai, I. H. (1992). Characterization and molecular-cloning of neurotoxic phospholipases- 
a(2) from Taiwan viper (Vipera-russelli-formosensis). European Journal of Biochemistry, 209, 635-641.

6. Arni, R. K., \& Gutierrez, J. M. (1993). Crystallization and preliminary diffraction data of two myotoxins isolated from the venoms of Bothrops asper (Terciopelo) and Bothrops nummifer (jumping viper). Toxicon, 31, 1061-1064.

7. Cintra, A. C. O., Marangoni, S., Oliveira, B., \& Giglio, J. R. (1993). Bothropstoxin-I-amino-acid-sequence and function. Journal of Protein Chemistry, 12, 57-64.

8. Lomonte, B., Angulo, Y., Rufini, S., Cho, W. H., Giglio, J. R., Ohno, M., et al. (1999). Comparative study of the cytolytic activity of myotoxic phospholipases $\mathrm{A}(2)$ on mouse endothelial (tEnd) and skeletal muscle (C2C12) cells in vitro. Toxicon, 37, $145-158$.

9. Swietnicki, W. (2006). Folding aggregated proteins into functionally active forms. Current Opinion in Biotechnology, 17, 367-372.

10. Paladini, A. A., Jr., \& Weber, G. (1981). Pressure-induced reversible dissociation of enolase. Biochemistry, 20, 2587-2593.

11. Silva, J. L., Foguel, D., Da Poian, A. T., \& Prevelige, P. E. (1996). The use of hydrostatic pressure as a tool to study viruses and other macromolecular assemblages. Current Opinion in Structural Biology, 6, 166-175.

12. Robinson, C. R., \& Sligar, S. G. (1995). Hydrostatic and osmotic pressure as tools to study macromolecular recognition. Methods in Enzymology, 259, 395-427.

13. Silva, J. L., \& Weber, G. (1993). Pressure stability of proteins. Annual Review of Physical Chemistry, 44, 89-113.

14. Lefebvre, B. G., Gage, M. J., \& Robinson, A. S. (2004). Maximizing recovery of native protein from aggregates by optimizing pressure treatment. Biotechnology Progress, 20, 623-629.

15. St John, R. J., Carpenter, J. F., \& Randolph, T. W. (1999). High pressure fosters protein refolding from aggregates at high concentrations. Proceedings of the National Academy of Sciences of the United States of America, 96, 13029-13033.

16. St John, R. J., Carpenter, J. F., \& Randolph, T. W. (2002). Highpressure refolding of disulfide-cross-linked lysozyme aggregates: Thermodynamics and optimization. Biotechnology Progress, 18, 565-571.

17. Foguel, D., Robinson, C. R., de Sousa, P. C., Jr., Silva, J. L., \& Robinson, A. S. (1999). Hydrostatic pressure rescues native protein from aggregates. Biotechnology and Bioengineering, 63, 552-558.

18. St John, R. J., Carpenter, J. F., Balny, C., \& Randolph, T. W. (2001). High pressure refolding of recombinant human growth hormone from insoluble aggregates. Structural transformations, kinetic barriers, and energetics. The Journal of Biological Chemistry, 276, 46856-46863.

19. Seefeldt, M. B., Ouyang, J., Froland, W. A., Carpenter, J. F., \& Randolph, T. W. (2004). High-pressure refolding of bikunin: Efficacy and thermodynamics. Protein Science, 13, 2639-2650.

20. Chura-Chambi, R. M., Genova, L. A., Affonso, R., \& Morganti, L. (2008). Refolding of endostatin from inclusion bodies using high hydrostatic pressure. Analytical Biochemistry, 379, 32-39.

21. Lee, S. H., Carpenter, J. F., Chang, B. S., Randolph, T. W., \& Kim, Y. S. (2006). Effects of solutes on solubilization and refolding of proteins from inclusion bodies with high hydrostatic pressure. Protein Science, 15, 304-313.
22. Schoner, B. E., Bramlett, K. S., Guo, H., \& Burris, T. P. (2005). Reconstitution of functional nuclear receptor proteins using high pressure refolding. Molecular Genetics and Metabolism, 85, 318-322.

23. Ward, R. J., de Oliveira, A. H., Bortoleto, R. K., Rosa, J. C., Faca, V. M., \& Greene, L. J. (2001). Refolding and purification of Bothropstoxin-I, a Lys49-phospholipase A2 homologue, expressed as inclusion bodies in Escherichia coli. Protein Expression and Purification, 21, 134-140.

24. Angulo, Y., \& Lomonte, B. (2005). Differential susceptibility of $\mathrm{C} 2 \mathrm{C} 12$ myoblasts and myotubes to group II phospholipase A2 myotoxins from crotalid snake venoms. Cell Biochemistry and Function, 23, 307-313.

25. Clark, E. D. B. (1998). Refolding of recombinant proteins. Current Opinion in Biotechnology, 9, 157-163.

26. Ami, D., Natalello, A., Taylor, G., Tonon, G., \& Maria Doglia, S. (2006). Structural analysis of protein inclusion bodies by Fourier transform infrared microspectroscopy. Biochimica et Biophysica Acta, 1764, 793-799.

27. Garcia-Fruitos, E., Aris, A., \& Villaverde, A. (2007). Localization of functional polypeptides in bacterial inclusion bodies. Applied and Environmental Microbiology, 73, 289-294.

28. Singh, S. M., \& Panda, A. K. (2005). Solubilization and refolding of bacterial inclusion body proteins. Journal of Bioscience and Bioengineering, 99, 303-310.

29. Patra, A. K., Mukhopadhyay, R., Mukhija, R., Krishnan, A., Garg, L. C., \& Panda, A. K. (2000). Optimization of inclusion body solubilization and renaturation of recombinant human growth hormone from Escherichia coli. Protein Expression and Purification, 18, 182-192.

30. da Silva Giotto, M. T., Garratt, R. C., Oliva, G., Mascarenhas, Y. P., Giglio, J. R., Cintra, A. C. O., et al. (1998). Crystallographic and spectroscopic characterization of a molecular hinge: Conformational changes in bothropstoxin I, a dimeric Lys49 phospholipase A2 homologue. Proteins, 30, 442-454.

31. Middelberg, A. P. (2002). Preparative protein refolding. Trends in Biotechnology, 20, 437-443.

32. Tsumoto, K., Umetsu, M., Kumagai, I., Ejima, D., Philo, J. S., \& Arakawa, T. (2004). Role of arginine in protein refolding, solubilization, and purification. Biotechnology Progress, 20, 13011308.

33. Prijatelj, P., Pranznikar, Z. J., Petan, T., Krizaj, I., \& Pungercar, J. (2008). Mapping the structural determinants of presynaptic neurotoxicity of snake venom phospholipases A(2). Toxicon, 51, $1520-1529$.

34. Aragao, E. A., Chioato, L., \& Ward, R. J. (2008). Permeabilization of $E$. coli $\mathrm{K} 12$ inner and outer membranes by bothropstoxin-I, A LYS49 phospholipase A2 from Bothrops jararacussu. Toxicon, 51, 538-546.

35. Leite, R. S., Giuliani, C. D., Lomonte, B., Franco, W., \& Selistrede-Araujo, H. S. (2004). Effect of a recombinant Lys49PLA2 myotoxin and Lys49PLA2-derived synthetic peptides from Agkistrodon species on membrane permeability to water. Toxicon, 44, 157-159.

36. Yang, W. L., Peng, L. S., Zhong, X. F., Wei, J. W., Jiang, X. Y., Ye, L. T., et al. (2003). Functional expression and characterization of a recombinant phospholipase A2 from sea snake Lapemis hardwickii as a soluble protein in E. coli. Toxicon, 41, 713-721. 